\title{
AN IN VITRO STUDY ON THE TOXIC EFFECTS OF NONYLPHENOLS (NP) IN MITOCHONDRIA
}

\author{
M. Bragadin ${ }^{1 *}$, G. Perin ${ }^{1}$, A.Iero, S. Manente, ${ }^{1}$ V. Rizzoli ${ }^{2}$ and G. Scutari ${ }^{2}$. \\ ${ }^{1}$ Dipartimento di Scienze Ambientali, Università di Venezia, DD 213730123 Venezia, Italy \\ ${ }^{2}$ Dipartimento di Chimica Biologica, Università di Padova, via Colombo 35121 Padova, Italy \\ (Received in Germany 15 June 1998; accepted 27 July 1998)
}

Correspondence to: Marcantonio. Bragadin*

Dipartimento di Scienze Ambientali, Università di Venezia DD 213730123 Venezia ITALY

tel. $+39+41+2578507 \quad$ Fax $+39+41+2578507 \quad$ Email: aleiero@unive.it

\section{Abstract}

This paper is focused on alkylphenols, compounds which are formed by the biodegradation of polyethoxilatedalkylphenols detergents. Our experiments show that alkylphenols act not only as detergents, but also as uncouplers of the oxidative phosphorylation. This effect, can be observed at very low doses, thus suggesting that the preferential target of nonylphenols in living organisms are mitochondria.

(C) 1999 Elsevier Science Ltd. All rights reserved

Introduction

The increasing use of detergents for industrial and household purposes is accompanied by studies on their biodegradability and toxicity in animals. To establish the molecular mechanisms responsible for the "in vivo" toxic effects of detergents, many studies have been performed in whole cells and in subcellular structures [16]. Less attention is devoted, in general, to the chemical compounds which are formed by biodegradation or chemical decomposition of the initial compounds. However, this is a fundamental problem since in many cases the decay products have more toxic effects than the initial compounds. In this paper we have analysed the molecular properties and the interactions with the mitochondrial membrane of Nonylphenols (NP). These compounds are generated by the biodegradation of polyethoxylatednonylphenols (NPEO) [7-9], a kind of detergent largely used in industries and for household purposes. Our results indicate that NP at very low doses inhibits the ATP synthesis in mitochondria. Therefore NPs like their parent NPEO, act not only as detergents, but also as very strong mitochondrial uncouplers.

Materials and Methods.

Mitochondria were prepared from the livers of fasted albino Wistar rats weighing about $300 \mathrm{~g}$. The livers were isolated and placed in ice-cold sucrose $0.25 \mathrm{M}$, Tris-Mops $1 \mathrm{mM}$, EGTA 0.1.M. The livers were rinsed 
three times with ice-cold medium, cut to small cubes with scissors, and passed through a Potter homogenizer fitted with a rotating teflon pestle kept in an ice--water bath (two strokes with $40 \mathrm{ml}$ of medium per liver). The homogenate was diluted to $200 \mathrm{ml}$ per liver, and unbroken cells and nuclei were sedimented by centrifugation at $650 \mathrm{xg}$ in a Sorvall RC2B refrigerated centrifuge kept at $2^{\circ} \mathrm{C}$ (rotor GSA at $2000 \mathrm{rev} . / \mathrm{min}$ for $10 \mathrm{~min}$ ). The supernatant was carefully decanted and centrifuged at $7700 \mathrm{xg}$ in the same centrifuge (rotor SS34 at $8000 \mathrm{rev} . / \mathrm{min}$. for $10 \mathrm{~min}$ ). The supernatant was disgarded, the mitochondria pellet was carefully resuspended in ice-cold isolation medium and spun as above. The resulting mitochondrial pellet was

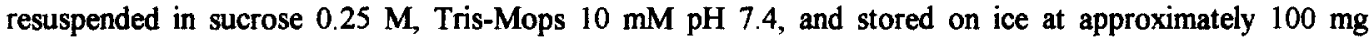
protein $/ \mathrm{ml}$. Mitochondrial protein was determined with the biuret reaction.Mitochondrial oxygen consumption was measured with a Clark oxygen electrode (Yellow Springs Instruments, OH, USA) fitted in a thermostat-controlled closed chamber equipped with magnetic stirring. The reaction medium $(2 \mathrm{ml})$ was kept thermostated at $25^{\circ} \mathrm{C}$ in all experiments. The rate of $\mathrm{K}^{+}$efflux was measured with a selective electrode (Beckman) in a glass vessel with a magnetic stirring. The recording apparatus was a Radiometer pH meter connected with a "Texas recorder". The mitochondria $(0.5 \mathrm{mg} / \mathrm{ml})$ were resuspended in a medium containing

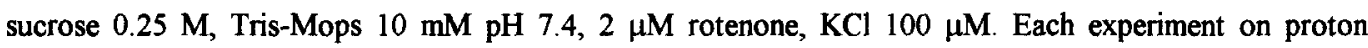
conductance was started with the addition of valinomycin (120 ng/mg protein) and, successively, of NP. Each experiment on potassium conductance was started with addition of FCCP $(2 \mu \mathrm{M})$ and NP All chemicals were of the highest purity commercially available, while NP and NPEO were a generous gift of $\mathrm{dr} A$. Orio. Valinomycin and FCCP were obtained from SIGMA, Milan.

\section{Results and discussion}

In the mitochondria, respiratory substrates produced in the Krebs cycle, reduce molecular oxygen and drive ATP synthesis. The reduction of oxygen occurs through a sequence of redox couples forming the respiratory chain (RC). The rate of oxygen reduction by the substrates (as shown in Fig. 1)

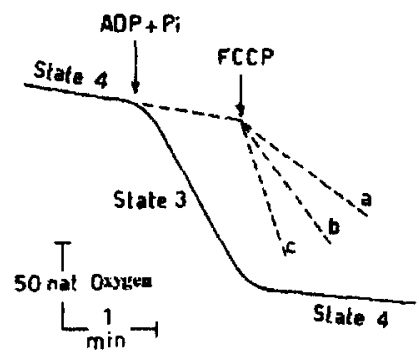

Fig.1 Mitochondrial Respiration In the presence of succinate $(2 \mathrm{mM})$, the respiratory rate of mitochondria (the ratio between oxygen concentration and time) is low and is named State 4 . In the presence of ADP and Phosphate $P_{i}$ the respiratory rate is enhanced (State 3) and after consumption of ADP and $P_{i}$, the rate returns in State 4 . In the presence of an uncoupler such as FCCP (broken line), the rate increases as the concentration of uncoupler is increased up to a maximim value. In trace a FCCP concentration is $0.6 \mu \mathrm{M}$, in $\mathrm{b}$ $7 \mu \mathrm{M}$, in c $8 \mu \mathrm{M}$. Medium composition: Sucrose $0.25 \mathrm{M}$. Tris-Mops $10 \mathrm{mM}$, Succinate $2 \mathrm{mM}$. Mitochondria : $1 \mathrm{mg} / \mathrm{ml}$. 
is low and this condition is named State 4 . The rate can be enhanced by addition of ADP and phosphate (Pi) to the system. In this condition (State 3, Fig.1) ATP synthesis occurs. After consumption of ADP, the respiratory rate returns to State 4 . The ratio between the rate in state 3 and in state 4 is defined Respiratory Control Ratio (RCR). In coupled mitochondria RCR, is about 6; when RCR=1, no ATP synthesis occurs [10]. The State 4 respiratory rate can also be enhanced in the presence of uncouplers. Uncouplers are chemical compounds which enhance the permeability of the mitochondrial membrane to the protons and collapse the electrochemical gradient $(\Delta \mathrm{pH}+\Delta \Phi)$ which drives ATP synthesis. Therefore in the presence of uncouplers, the $\mathrm{RC}$ reaches the maximum value (in the presence of $2 \mu \mathrm{M} \mathrm{FCCP*}$, the respiratory rate is.about $150 \mathrm{n}$ at oxygen/mg prot $/ \mathrm{min}$ ) and no ATP synthesis occurs.

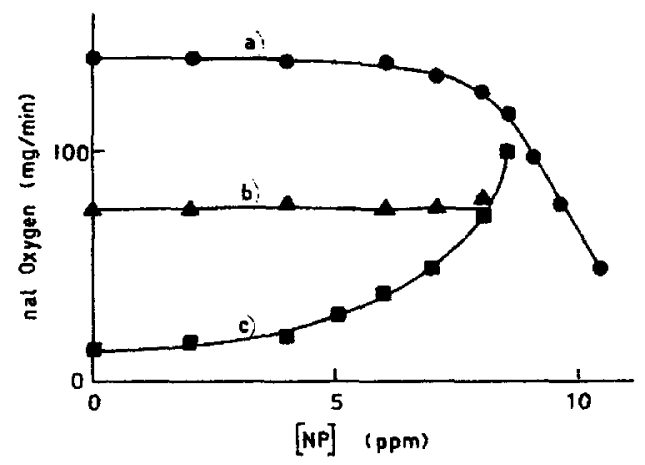

Fig. 2 Interaction of NP with mitochondrial respiration.

When the mitochondria are uncoupled by FCCP, the trace a) shows the inhibitory effect of increasing amounts of NP. NP inhibits the maximal respiratory rate above $9 \mathrm{ppm}$. The State 3 respiratory rate b) is not influenced by NP. The state 4 respiratory rate c) is enhanced by NP. This behaviour is typical of uncouplers. When the ratio between state 3 and state 4 is one, no ATP synthesis occurs. This situation is obtained with 8 ppm NP. Medium composition as in Fig.1.

Fig. 2 shows the effects of increasing amounts of NP on mitochondrial respiration. The results indicate that State 3 respiration is not modified, thus demonstrating that NP does not interfere with Pi, ADP and ATP carriers. The most significant effect is an enhancement of State 4 respiratory rate. At about $8 \mathrm{ppm} \mathrm{NP}$, the ratio between State 3 and State 4 is one: this means that no ATP synthesis and cell death results.

The enhancement of State 4 is generally induced by uncouplers which increase the membrane permeability to protons; this behaviour however, can also be induced by detergents which enhance membrane permeability to all ions, including the protons. Following a procedure suggested by Bragadin et al. (paper submitted), to determine whether the enhancement of State 4 is due to an uncoupling or to a detergent effect, we have operated as follows: mitochondria contain $0.1 \mathrm{M}$ potassium in the internal matrix. If non respiring mitochondria are resuspended in a potassium-free medium, no potassium efflux is detected by a selective potassium electrode. A potassium efflux is monitored only in the presence of Valinomycin, a well known potassium carrier, and provided that a counter ion enters the matrix. The counterion may be a proton if a proton carrier (an uncoupler) is present. In this case, operating with an excess of Valinomycin, the rate 
limiting step of the whole kinetic is the proton influx, the rate of which is proportional to the concentration of uncoupler.

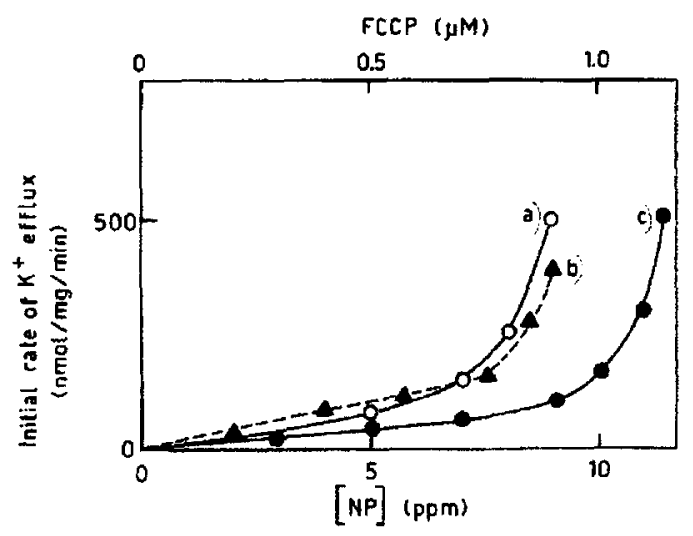

Fig. 3 Measurements of mitochondrial membrane conductance to protons a) $(\mu), b)(\sigma)$ and to potassium c) $(\lambda)$. In trace a), non respiring mitochondria $(0.5 \mathrm{mg} / \mathrm{ml})$ are resuspended in a medium with low potassium concentration (medium composition: sucrose $0.25 \mathrm{M}$, Tris-Mops $10 \mathrm{mM}, \mathrm{MgCl}_{2} 2 \mathrm{mM}$ and $\mathrm{KCl} 100 \mu \mathrm{M}$ ). The initial rate of potassium efflux is monitored by addition of an excess of valinomycin ( $2 \mathrm{n}$ moles $/ \mathrm{mg}$ protein) and varying amounts of NP. In this case since valinomycin is in excess, the rate limiting step is the proton conductance due to NP and therefore the trace a) reports the proton conductance caused by NP $(\mu)$. The broken line b) $(\sigma)$ reports the initial rate of potassium efflux in the presence of varying amounts of the uncoupler FCCP and in excess of valinomycin. Trace $c)$ is the potassium conductance ( $\lambda$ ), since the trace reports the potassium efflux in the presence of excess FCCP and varying amounts of NP. In this condition since the uncoupler FCCP is in excess, the rate limiting step is the potassium transport caused by NP c).

Fig. 3 (trace b) reports the initial rate of potassium efflux which is proportional to the concentration of the uncoupler FCCP. As shown in this figure, the same behaviour is obtained at varying amounts of NP (Fig.3, trace a). NP behaves therefore as an uncoupler by increasing the membrane permeability to the protons. This is not unespected since NP is a phenol and some uncouplers are phenols [1]. The same procedure may be utilized to establish if a compound enhances the permeability of a membrane to potassium. In this case, an excess of uncoupler is added to the suspension of non respiring mitochondria and, in the presence of a compound which enhances potassium permeability, an outward potassium flow is monitored. This is the case of NP (Fig.3, trace c). This experiment demonstrates, therefore, that NPs enhance the potassium permeability. This behaviour is typical of detergents [1], and demonstrates that NP is a detergent. Therefore NP is also a detergent. If however we compare the diagrams of Fig. $3 a$ and $3 c$, we can verify that NP is more effective as an uncoupler, since at the same dose the effect is stronger on proton conductance than on potassium. This means that the preferential target of NP is to the mitochondrial membrane and ATP synthesis in particular, which is inhibited or reduced. The same general trend is true for all structures correlated with ATP utilisation. In this respect, therefore, toxicological consequences due to NP are similar to those typical of uncouplers. For comparison, we have observed that the parent chemical compound NPEO does not affect any function of mitochondria prior to biodegradation (data not shown). We conclude that with regard to ATP synthesis, the biodegradation product NP is much more toxic than the parent chemical compound NPEO. *FCCP = carbonylcyanide-p-trifluoromethoxyphenyl hydrazone 


\section{References}

1. M. Bragadin and P. Dell'Antone, Mitochondrial Bioenergetics as affected by cationic detergents, Arch. Environ. Contam. Toxicol., 30, 280-284 (1994)

2. M. Bragadin, G. Perin, S. Raccanelli and S. Manente, The accumulation in Lysosomes of the Anionic Detergent Linear Alkylbenzene Sulphonate (LAS) Arch. Environ. Contam. Toxicol. 15, 1749-1751 (1996)

3. G.A. Blondin, L.M. Knabeloch, H.W. Read and J.M. Arkin, Mammalian Mitochondria as "in vitro" monitors of water quality, Bull. Emviron. Contam. Toxicol. 38, 467-474 (1987)

4. R.D. Shannon, G.D.Boarman, A.M. Dietrich and D.R. Bevan, Mitochondrial response to chlorophenols as a short-term toxicity assay, Environ. Toxicol. Chem. 10, 57-66 (1991).

5. M. Bragadin and $P$. Dell'Antone, A New In Vitro toxicity test based on the response to toxic substances in solutions of mitochondria from beef heart, Arch. Environ. Contam. Toxicol. 27, 410-414 (1994).

6. B. Fell, Tenside: Aktueller Stand-Absehbare Entwicklungen, Tenside Surf. Det, 28, 385-395 (1991).

7. W. Giger, P.H. Brunner and C. Schaffer, Nonylphenol in Sewage Sludge: Accumulation of Toxic Metabolites from Nonionic Surfactants, Science (Washington D,C.) 225, 623 (1984).

8. W. Giger, M. Ahel, H.U. Coch, C. Laubscher, C. Schaffner and J. Schneider, Behaviour of Alkylphenol Polyethoxylate Surfactants and of Nitrilotriacetate in Sewage Treatement, Water Sci. Technol. 19, 449460 (1987).

9. M. Ahel, W. Giger and T. Konrad, Persistent Organic Chemicals in Sewage Effluents. Determinations of Nonylphenoxy Carboxylic Acids by high-resolution Gas Chromatography Mass Spectrometry and High performance liquid Chromatography, Environ. Sci. Technol. 21, 697-703 (1987).

10.R.W. Estabrook, Mitochondrial Respiratory control and the Polarographic Measurement of ADP:O Ratios. in Methods in Enzymology Vol X Edited by Ronald W. Estabrook, Maynard E. Pullman Academic Press New York and London p. 47 (1967).

11.M. Stockdale and M. Seldwin, Effects of ring substituents on the activity of phenols as inhibitors and uncouplers of mitochondrial respiration, Eur. J. Biochem. 21, 565-574 (1971). 